\title{
Discussion on Continuous Airworthiness and Maintenance of Civil Aircraft
}

\author{
Sai Luo* \\ Maintenance Support Department of Jinan International Airport, Jinan 250102, Shandong Province, China \\ *Corresponding author: Sai Luo, hkkiol@sina.com
}

Copyright: () 2022 Author(s). This is an open-access article distributed under the terms of the Creative Commons Attribution License (CC BY 4.0), permitting distribution and reproduction in any medium, provided the original work is cited.

\begin{abstract}
With China's rapid economic development, the civil aviation market's development area has expanded. On this basis, the state has proposed more extensive and stringent civil aviation rules. Many factors can affect the civil aircraft during the real operation process, and this can have an impact on the aircraft's regular functioning to some level. To ensure that civil aircraft remain airworthy, appropriate maintenance work must be performed on a regular basis to prevent compromising the aircraft's safety performance. For the purpose of reference, this paper discusses the continual airworthiness and maintenance of civil aircraft.
\end{abstract}

Keywords: Civil aircraft; Continuous airworthiness; Repair

Online publication: January 18, 2022

\section{Introduction}

According to the current state of the civil aviation sector, there have been several flight accidents caused by inappropriate maintenance of civil aircraft both at home and abroad in recent years, resulting in largescale property losses and deaths. In reality, numerous external factors can have an impact on the functioning of civil aircraft. It is vital to carry out scientific and reasonable maintenance to promote the continuing normal functioning of aircraft in order to improve its performance during operation.

\section{Concept of continuous airworthiness of civil aircraft}

Civil aircraft airworthiness is a standard that ensures the safety of aircraft while in flight, and it is separated into "initial airworthiness" and "continuous airworthiness." Initial airworthiness means that all parts of the aircraft must be manufactured in accordance with current airworthiness standards at the start of the design process, whereas continuous airworthiness means that after the aircraft has received airworthiness approval from the national department, the entire process from putting it into use to scrapping must be followed, and appropriate maintenance work must be carried out during the application process to ensure the safety and stability of its operation. One of the most significant qualities of a civil aircraft is its continuous airworthiness; that is, the civil aircraft should function normally within the scope authorized by the state, and its parts and design should conform with applicable rules.

\section{Maintenance management of civil aircraft}

The purpose of maintenance management for civil aircraft is to provide guarantee for the safety and stability of its use process. Under normal circumstances, the maintenance management mainly contains the corresponding technical performance and specific rules, the implementation of maintenance management, 
from the manufacturing structure, maintenance methods, principles of use, design principles and other aspects, to carry out scientific and reasonable management. Before carrying out maintenance on aircraft, its use principle should be fully considered first, because maintenance work must run through the whole use process of aircraft, so it is necessary for relevant maintenance management units to carry out regular audit on the repaired aircraft and confirm that the audit is passed before obtaining the certificate of conformity. Only then can the aircraft be reasonably used within the effective range. Therefore, we must actively improve the management effect of continuous airworthiness and the corresponding maintenance level, so as to lay a foundation for the safe application of aircraft ${ }^{[1]}$.

In the process of aircraft application, the natural environment or failure can make it deviate from the airworthiness standard. In this case, the deviation must be corrected in a timely manner by means of maintenance, so as to ensure that the aircraft can be in a normal and stable operation state. Moreover, in the process of aircraft operation, a certain degree of wear is bound to occur, including parts aging, tire wear, etc. all aspects of wear can cause the degradation of aircraft performance, and even directly lead to mechanical failure. Moreover, the longer the aircraft is put into operation, the greater the possibility of failure. At the initial stage of design, the performance of aircraft is completely determined by design and manufacturing, so its quality can have a very important impact on the effect of later maintenance work. Under normal circumstances, the purpose of the design and production of various types of aircraft is to effectively meet a variety of use needs. For different types of aircraft, the maintenance methods applied in the maintenance process are also different. However, during the maintenance, the work shall be carried out strictly according to the aircraft's own performance, so as to promote the timely clarification of airworthiness information, and be able to reasonably design the maintenance scheme according to the actual situation to ensure the good airworthiness of the aircraft.

\section{Main contents of continuous airworthiness and maintenance of civil aircraft}

On the basis of the continuous expansion of the scale of airlines, the maintenance of civil aircraft is becoming more and more difficult, so it is necessary to appropriately increase the corresponding maintenance investment. Turbofan engine aircraft have the largest application range among civil aircraft. It works on the principle of supplemental energy and fluid energy created by machine rotation. The circulating components primarily consist of a high-pressure compressor, a low-pressure compressor, a highpressure turbine, a low-pressure turbine, a fan blade, and a combustion chamber, with the high-pressure turbine, combustion chamber, and the high-pressure compressor referred to as the "core engine," which can discharge gas and generate a certain thrust, allowing the aircraft to operate normally.

Overall, the maintenance management system for turbofan engine aircraft is highly complicated, and its airworthiness management is strongly tied to many sectors. It is important to conduct various management and supervision on its airworthiness from a variety of perspectives, including "person, machine, material, technique, and environment." For example, collecting and sorting data is a crucial part of everyday maintenance labor. On this basis, if the maintenance department has difficulties with its job, the airworthiness department can act quickly. Furthermore, when the airworthiness department's maintenance work is finished, it should be inspected at least once to give essential assurance for the aircraft's operation status ${ }^{[2]}$.

\subsection{Continuous airworthiness standard}

From a technical point of view, the main purpose of airworthiness standard is to provide guarantee for the minimum safety standard in the operation of civil aircraft. Compared with other types of standards, airworthiness standard is a national standard with strong clarity. At present, its corresponding government departments and relevant authorized management departments have put forward corresponding regulations, 
and the basis of the regulations is the safety of civil aircraft. Moreover, its corresponding laws and regulations have been incorporated into the maintenance management system of civil aircraft. In this process, in order to effectively ensure flight safety and minimize aircraft operation costs, the formulation of airworthiness standards has specific and pragmatic characteristics, which can facilitate the continuous operation of aircraft. Moreover, the birth background of most airworthiness standards is several previous air accidents, so scientific and reasonable airworthiness standards for civil aircraft are of great practical significance.

\subsection{Main maintenance methods}

During the maintenance of civil aircraft, reasonable maintenance methods shall be selected according to the actual situation. Generally speaking, the applicable maintenance methods include planned / unplanned maintenance and aircraft flight status monitoring. The above different maintenance methods are based on the corresponding airworthiness standards of turbofan engine aircraft. In particular, maintenance standards such as aircraft flight status monitoring must be based on the aircraft's own flight status and parameters. Only through scientific and reasonable detection of the aircraft can we have a fuller understanding of all aspects of the situation, and if there are problems, they can also be clarified and repaired in time, so as to reduce the flight risk of the aircraft as much as possible. At the same time, planned maintenance is to carry out comprehensive and detailed maintenance for the aircraft within a certain time range to ensure that the aircraft can meet the existing design requirements after maintenance. Planned maintenance usually takes a long time, but the degree of maintenance is relatively deeper. Finally, unscheduled maintenance is mainly applied in case of aircraft failure. Although this maintenance mode has low cost, it has greater risk. Therefore, the maintenance unit needs to reasonably select the maintenance mode based on the actual operation of the aircraft ${ }^{[3]}$.

\subsection{Security system}

When carrying out maintenance work for turbofan engine aircraft, it shall be comprehensively evaluated according to different technical standards to ensure that the maintenance process, methods and ways are consistent with the corresponding technical standards. At the same time, the maintenance unit shall carry out real-time management and supervision for the maintenance process. In case of failure in the aircraft, the maintenance personnel shall immediately carry out detailed detection. If the maintenance personnel violate the rules in this process, it will affect the continuous airworthiness of the aircraft to a certain extent and may cause safety problems. However, in fact, some staff are prone to negligence during maintenance, resulting in more serious potential safety hazards during aircraft operation. In addition, according to relevant statistics, the proportion of human factors in accidents caused by aircraft has increased significantly in recent years, so the maintenance of civil aircraft is currently carried out. It is necessary to reconstruct and build the corresponding safety system to effectively control human errors in the maintenance process.

\subsection{Continuous airworthiness and maintenance management}

For maintenance units, they should pay attention to improving their own level of aircraft management and maintenance. Therefore, relevant staff are required to continuously strengthen their theoretical knowledge on civil aircraft safety, promote their professional skills, and regularly carry out education and training. By strengthening the awareness of relevant staff on the significance of the continuous airworthiness of civil aircraft and providing more power for them to carry out in-depth research on the continuous airworthiness of aircraft, relevant staff can effectively avoid mistakes in the work process, and then improve the maintenance quality and the safety of the maintenance process, which can effectively reduce the risk of aircraft in operation. In addition, airworthiness management department also needs to reasonably manage 
and supervise the maintenance of aircraft, so as to ensure the continuous airworthiness and flight safety of aircraft ${ }^{[4]}$.

\section{Airlines and airworthiness safety}

In reality, difficulties in aviation maintenance work include low technical requirements for airline work, which can easily lead to maintenance personnel's neglect during the work process or inability to follow norms and regulations. Simultaneously, regular scheduled maintenance can easily lead to tiredness among important staff, compromising the maintenance impact.

To carry out maintenance management among airlines, first of all, we need to pay attention to the cultivation of airworthiness safety ideology, so that relevant staff can gradually change their work consciousness from initial "want me to be airworthy" to active "I want to be airworthy." At the same time, we also need to take "sustainable airworthiness" as the core, carry out education and training, and effectively implement relevant documents, any mistakes in this work are strictly prohibited. Furthermore, strict management shall be carried out for relevant maintenance personnel. When carrying out maintenance work, the staff must be authorized to work and carry out maintenance operations, and the identity certificates used in the work process must be managed by special personnel and cannot be distributed at will. In the whole process of work, relevant departments need to provide scientific and reasonable supervision and management to ensure the effective implementation of all aspects of work ${ }^{[5]}$.

\section{Conclusion}

In general, the operation impact of civil aircraft may be influenced not only by ongoing airworthiness, but also to some part by maintenance. As a result, in order to keep civil aircraft in normal operation, it is required to choose a maintenance mode that is appropriate for the aircraft's ongoing airworthiness and maintenance concept. At the same time, maintenance personnel must focus entirely on their own job and strive to improve the safety of the maintenance process as much as possible. In order to successfully increase the continuous airworthiness level and maintenance impact of civil aircraft, relevant departments must provide reasonable monitoring and management during this process.

\section{Disclosure statement}

The author declares no conflict of interest.

\section{References}

[1] Cui G, 2021, Discussion on Risk Management Strategy in Civil Aircraft Maintenance. (22): 279.

[2] Su X, He Q, Zhang B, et al., 2020, A Comprehensive Maintenance Platform for Civil Aircraft Brake Device, CN210210316U[P].

[3] Li Z, Zhang W, Jiang S, 2019, Analysis of Qualitative Risk Assessment Method for Continuous Trial Flight of Civil Aircraft. Science and Technology Innovation Herald, 16(07): 20-21.

[4] Xiao J, Huang C, Liu Y, 2019, Research on Continuous Airworthiness of Domestic Civil Helicopter. China Flights, 000(013): 1-3.

[5] Huang F, 2021, Analysis on Pilot Work of Aircraft Maintenance Professional "N+1" License Training Model. (10): 87-89. 